\title{
Processi di radicalizzazione e prospettive trasformative
}

\author{
di Loretta Fabbri^, Alessandra Romano ${ }^{\circ *}$
}

\section{Riassunto}

Il contributo esplora le azioni di ricerca-formazione sui processi di polarizzazione e radicalizzazione, promosse all'interno del Progetto Forward (MUR ID 85901). Ricostruisce il framework teorico-concettuale del progetto, approfondendo, nello specifico, il contributo della teoria trasformativa (Mezirow, 2003; Amiraux \& Fabbri, 2020; Sabic-El-Rayess \& Marsick, 2021) e degli approcci intersezionali (Crenshaw, 2017; Colombo, 2020) nella comprensione dei processi di radicalizzazione. Un'attenzione specifica è rivolta all'impianto metodologico e all'articolazione dei sistemi di attività realizzati all'interno del Progetto Forward, che comprendono la costruzione di un network di esperti internazionali sui processi di radicalizzazione, le azioni di ricerca-formazione con i professionisti che hanno contatti con cittadini provenienti da altri paesi, e il lancio di un centro internazionale di studi sulla microradicalizzazione.

Parole chiave: radicalizzazione; apprendimento trasformativo; intersezionalità; ricerca-formazione; progetto FORwARD.

\section{Processes of radicalization and transformative perspectives}

\begin{abstract}
The contribution explores the research-training actions on processes of polarization and radicalization, promoted within the Forward Project (MUR

\footnotetext{
${ }^{\wedge}$ Università degli Studi di Siena.

○Università degli Studi di Siena. Corresponding author: alessandra.romano2@unisi.it.

* Il contributo è frutto dei continui scambi delle due Autrici. Solo per ragioni di responsabilità scientifica, si specifica che Loretta Fabbri è Autrice del Paragrafo "Il Progetto FORwARD (MUR ID 85901)", Alessandra Romano è Autrice del Paragrafo "La prospettiva trasformativa come framework interpretativo della radicalizzazione", ed entrambe hanno curato la stesura del Paragrafo "Università in apprendimento".
}

Educational Reflective Practices (ISSNe 2279-9605), 1/2021 Special Issue Doi: 10.3280/erp1-special-2021oa12448 
ID 85901). It reconstructs the theoretical-conceptual framework of the project, specifically investigating the contribution of transformative theory (Mezirow, 2003; Fabbri \& Amiraux, 2020; Sabic-El-Rayess \& Marsick, 2021) and intersectional approaches (Crenshaw, 2017; Colombo, 2020) in understanding radicalization processes. Specific attention is paid to the methodological framework and the articulation of the systems of activities implemented within the Forward Project, which include the construction of a network of international experts on radicalization processes, researchtraining actions with professionals who have contact with citizens from other countries, and the launch of an international center for micro-radicalisation studies.

Keywords: radicalization; transformative learning; intersectionality; research-training; FORwARD project.

First submission: 01/09/2021, accepted: 13/09/2021

Available online: 30/09/2021

\section{Il Progetto FORwARD (MUR ID 85901)}

Il contributo esplora le azioni di ricerca-formazione promosse all'interno del progetto triennale "FORwARD - Formazione, ricerca e sviluppo di strategie "Community Based" per facilitare e supportare le pratiche di convivenza nei contesti multietnici"1.

Il progetto FORwARD si colloca all'interno del mandato definito dalla committenza ministeriale: "costituire reti universitarie in attuazione di accordi di collaborazione tra le università italiane e quelle degli stati aderenti all'organizzazione della cooperazione islamica". Il progetto si è tradotto nell'attenzione all'attivazione di ricerche-formazione secondo la prospettiva che vede apprendimento-ricerca-formazione come pratiche intersezionali (Crenshaw, 2017), finalizzate a tematizzare la complessità degli elementi che caratterizzano la radicalizzazione. Si sono così promossi dispositivi che hanno consentito sia all'università che agli attori organizzativi coinvolti di entrare in un processo di inquiry per la promozione di azioni capaci di suggerire contatti ad alto tasso di inclusività e contenere lo sviluppo di prospettive di significato distorte.

\footnotetext{
1 Finanziato dal MUR (ID 85901). Per ulteriori informazioni: https://www.forwardproject.unisi.it/.
} 
In origine, il mandato del progetto FORwARD è stato formulato all'interno di un'attenzione marcata ai crescenti fenomeni di radicalizzazione manifestatisi in molte aree del mondo dalle tragiche vicende degli attentati del 2001. Si pensi agli attacchi terroristici di Madrid (2004), Londra (2005), Parigi (2015), Bruxelles (2016), solo per citarne alcuni rivendicati da falangi estreme di matrice religiosa.

L'analisi e la ricerca-intervento sui processi di radicalizzazione sono ad oggi ancora più emergenti e urgenti: il caso Afghanistan apre questioni che non riguardano solo le politiche internazionali, ma le pratiche di apprendimento e disapprendimento che caratterizzano i sistemi di significato in gioco (Fabbri \& Melacarne, 2020). Quali categorie sono utili per analizzare la frammentarietà e l'instabilità della situazione afghana?

Questi eventi rendono ancora più importante "stare sul problema" della gestione delle differenze nei diversi contesti, non solo multietnici, ma anche entro gli stessi contesti di appartenenza territoriale.

La prospettiva che abbiamo adottato, in linea con la letteratura internazionale, è di interpretare i processi di radicalizzazione come processi di apprendimento e disapprendimento, che possono essere caratterizzati da categorie distorte, apprese inconsapevolmente nei contesti di vita quotidiana. La radicalizzazione non ha a che fare solo con atti terroristici, ma con pratiche sociali e culturali o traumi psicologici che sviluppano forme di pensiero tendenzialmente contraddistinte da polarizzazioni, teorie del complotto, mancato senso di appartenenza (Doosje et al., 2016).

$\mathrm{Ci}$ siamo posizionati dentro la filiera degli studi sull'apprendimento adulto che indagano come e a quali condizioni l'apprendimento può produrre cambiamento delle prospettive di significato in individui, gruppi e comunità ad alto tasso multietnico e a rischio di radicalizzazione (Amiraux \& Fabbri, 2020). In questo caso, la teoria trasformativa viene utilizzata come dispositivo da attivare nella formazione e negli apprendimenti informali ${ }^{2}$.

Gli eventi che stanno accadendo in Afghanistan nel 2021 rendono ingenue quelle letture che collocano la diversità a partire dalle diverse apparte-

\footnotetext{
${ }^{2}$ Nella letteratura internazionale, il costrutto di polarizzazione indica il meccanismo di pensiero che individua in polarità contrapposte la modalità di costruzione dell'argomentazione. È un automatismo cognitivo che utilizza categorie interpretative dicotomiche per fenomeni, oggetti, eventi complessi, e che, attraverso tendenze semplificatorie, riporta la costruzione di processi interpretativi a categorie contrapposte (Sabic-El-Rayess \& Marsick, 2021). Si pensi, per esempio, a chi dichiara di essere contro l'accoglienza degli immigrati perché questi metterebbero a rischio l'identità nazionale, oppure a chi guarda con sospetto il vaccino per il Covid-19 ipotizzando complotti sovranazionali per imporre dei comportamenti alla popolazione. Sono solo alcuni degli esempi di distorsioni cognitive ed epistemologiche esito di meccanismi di ragionamento polarizzati.
} 
nenze territoriali e impongono l'attenzione sulla difficoltà da parte delle comunità di apprendere sistemi di azione diversi da quelli adottati che si rivelano disfunzionali. Il piano micro diventa un piano di intervento educativo.

Processi di polarizzazione, radicalizzazione, l'adozione di teorie complottistiche sono elementi che vengono appresi e con cui gli individui costruiscono le prospettive di significato con cui interpretano il mondo. Come costruire delle arene discorsive dove le tesi diventano oggetto di validazione, dove si possono intravedere prospettive di significato diverse in grado anche di correggere o di contenere le distorsioni presenti?

Quello che dalla ricerca emerge è che gli individui sono più propensi ad identificarsi con una categoria sociale disponibile, come quella fornita da un gruppo o un'ideologia radicale (Sabic-El-Rayess \& Marsick, 2021) che, con la sua visione estrema, chiara ed esplicativa del mondo risponde al bisogno epistemico di avere un senso di certezza su di sé e sugli eventi del mondo. I processi di socializzazione all'interno di gruppi monoideologici possono alimentare un effetto definito a "camera d'eco" (Geeraerts, 2012), per cui le opinioni polarizzate e distorte si rafforzano a vicenda e diventano più estreme.

I meccanismi di ragionamento polarizzati e dicotomici rafforzano sentimenti di alienazione, impotenza e ostilità verso la società, sfiducia verso $\mathrm{i}$ metagaranti istituzionali, aumentando l'accettazione dell'uso della violenza e diminuendo il sostegno alle azioni democratiche (Lamberty \& Leiser, 2019), agendo come "moltiplicatori di radicalizzazione" (Byington, 2019). A fronte di questo quadro, come e a quali condizioni si "spacchetta" e "smonta" un pensiero radicalizzato che insiste su meccanismi di ragionamento polarizzati? Qual è il contributo delle teorie dell'apprendimento informale e trasformativo nel comprendere (e nell'intervenire su) processi di radicalizzazione in atto nei quartieri, nelle scuole, nelle istituzioni? Queste sono alcune delle domande di indagine che abbiamo affrontato all'interno del Progetto FORwARD.

\section{Università in apprendimento}

Il progetto FORwARD ha previsto nella prima annualità la promozione di un confronto tra università italiane (Università per Stranieri di Siena) e università straniere (Università Al Kawahayn, Università Al-Quaraouiyine di Fès, Marocco, University of Georgia, Teachers College, Columbia University, USA, Université de Montréal, Canada). Abbiamo aperto una dialettica con studiosi che avevano condotto studi e ricerche e azioni trasformative 
sul tema della radicalizzazione come costrutto che si apprende nei diversi contesti sociali.

Si sono pianificati un ciclo di incontri finalizzati al confronto e all'analisi di dilemmi disorientanti, incidenti critici, pratiche promettenti in atto nei diversi paesi. Eravamo consapevoli che eravamo di fronte a problemi che non necessariamente hanno trovato soluzione, o per cui è possibile trovare una soluzione.

Abbiamo implementato confronti transdisciplinari tra scienziati sociali provenienti da diverse aree culturali, esperienze, ambiti disciplinari, ma tutti impegnati a tematizzare una società ad alto tasso multietnico, hanno condotto il discorso scientifico su framework intersezionali (Crenshaw, 2017; Colombo, 2020). Si tratta di oggetti che non hanno appartenenza disciplinare, perché risiedono in un territorio i cui confini non sono dati ma negoziati. L'approccio intersezionale, tematizza che razzismo, abilismo e sessismo non sono forme di discriminazioni separate: per questo, considera gli effetti che l'intersecarsi tra forme di discriminazioni multiple (per esempio, per genere ed etnia, o per genere e disabilità, etc.) può avere sulla storia, sui corpi e sulle esperienze soggettive di individui, gruppi e comunità (Crenshaw, 2017). Le prospettive intersezionali (Crenshaw, 2017) indagano gli effetti derivanti dalla combinazione di più dimensioni di diversità (intersectionality, YuvalDavis, 2006), e i meccanismi di oppressione cumulativa legata a condizioni considerate di «duplice svantaggio» (Romano, 2020a, p. 29) ${ }^{3}$. I processi di radicalizzazione che adottano le donne occidentali e i processi di radicalizzazione che subiscono le donne afghane chiamano in causa diverse condizioni di fragilità. L'intersezionalità consente ai ricercatori e alle ricercatrici di diventare consapevoli che si muovono in territori molto diversi da ricercatrici e ricercatori nere, il cui colore della pelle diventa il tratto saliente per la discriminazione, da ricercatori e ricercatrici che lavorano in università in paesi non occidentali e dalla forte instabilità politica, dove la questione di genere diventa un teatro di scontro e di emarginazione (Colombo, 2020). La prospettiva degli studi intersezionali delinea gli strumenti necessari per accrescere la voce e il potere dei gruppi storicamente discriminati nel giogo

\footnotetext{
${ }^{3}$ Secondo quest'approccio, le fonti della disuguaglianza non sono sullo stesso livello. A seconda del livello di visibilità della diversità (il genere o il colore della pelle non può essere nascosto come, per esempio, l'orientamento sessuale), possibilità di scelta (una persona non può scegliere la propria età come può scegliere, invece, la confessione religiosa professata), sistema di regolazione normativa e legislativa (le leggi offrono garanzie contro le discriminazioni, tutele su alcune dimensioni di diversità, ma non su altre, e ciò che resta tagliato fuori dalla regolamentazione normativa, produce comunque disuguaglianze o discriminazioni), cambiano le opportunità (e gli ostacoli) che le persone possono incontrare nel loro percorso di affermazione e di autorealizzazione (Romano, 2020a).
} 
coloniale occidentale, contestando i gruppi dominanti (maschi, bianchi, europei), mettendo in discussione le loro regole (Spivak, 1999). Tematizza i modi in cui si costruisce politicamente e socialmente la supremazia dei maschi bianchi, come si difendono e si perpetuano i loro privilegi (Colombo, 2020).

L'ancoraggio agli approcci intersezionali ha consentito di apprendere che i processi di radicalizzazione, per esempio, costituiscono una realtà "diffusa e puntiforme", estremamente eterogenea in ogni sua sfaccettatura e in costante evoluzione. Qualsiasi visione disciplinarmente orientata corre il rischio di riportare entro categorie semplificative costrutti e oggetti complessi (Colombo \& Rebughini, 2016). Interessarsi di radicalizzazione significa, quindi, non guardare solo a stati e nazioni da sempre interessati da scontri tra fazioni opposte per ideologie di matrice religiosa (e che con gli stati occidentali hanno poco a che vedere). Esistono processi di pensiero radicalizzati e forme di polarizzazioni antinomiche anche all'interno della stessa nazione e su questioni che hanno a che fare con la salute pubblica, con l'emergenza pandemica, con la credibilità delle istituzioni politiche e governative.

Più i problemi sono complessi e non di facile risoluzione, o con soluzioni a bassa definizione e quindi open-ended, più c'è bisogno che la ricerca scientifica diventi una pratica socialmente condivisa, che coinvolge attori, comunità, organizzazioni. Quando si parla di apprendimento trasformativo, si parla della trasformazione delle prospettive di significato e questa non può avvenire se non attraverso il coinvolgimento e la consapevolizzazione dei soggetti coinvolti. Freire (1971), per esempio, è tra le fonti della teoria trasformativa, come portatore di un'idea di pratiche di apprendimento come forma di emancipazione rispetto a visioni della vita riduttivistiche o disfunzionali (Mezirow, 2003).

Come referenti del progetto, abbiamo proceduto alla traduzione del mandato formale, aprendo tre sistemi di attività:

1. la formazione dei professionisti che hanno contatti con cittadini provenienti da altri paesi - appartenenti a contesti sanitari, carcerari, scolastici - con l'obiettivo di supportarli nel validare gli apprendimenti pregressi e le prospettive distorte (per esempio, le regole sono uguali per tutti, i protocolli sanitari sono universali, la presenza di alunni stranieri in aula non consente di svolgere il programma ministeriale, i quartieri sono invasi da stranieri che disturbano la quiete pubblica, eccetera);

2. un setting di sviluppo professionale di practitioner configuratosi come master, in grado di lavorare sulla validazione delle prospettive in gioco e la conseguente individuazione delle distorsioni;

3. la costruzione di un centro internazionale di studi sui processi di microradicalizzazione e di quella forma di radicalizzazione cognitiva e diffusa, 
che non si traduce in atti terroristici ma in visioni polarizzate e complottistiche dei rapporti sociali, di lavoro e delle relazioni.

Partendo dal presupposto che ricerca, formazione e apprendimento sono processi difficilmente distinguibili, dentro un paradigma post-qualitativo, lo studio di un quartiere di una città di provincia che in pochi anni diventa un quartiere multietnico, aprendo forme di reazione difensive, ha rappresentato

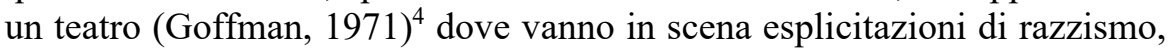
talvolta inconsapevole, che si sono tradotte in azioni organizzate attraverso l'istituzione di comitati di quartiere il cui ruolo è quello di difendersi da presunte minacce provenienti dalla presenza di tanti "stranieri".

Al contempo, abbiamo intercettato quelle forme di contatto che nascono attraverso la frequentazione di negozi gestiti da persone alloctone, l'interazione tra le madri nei giardini pubblici, fanno intravedere quegli apprendimenti informali che nascono da contatti occasionali, da relazioni improvvisate che fanno nascere dubbi sulla necessità di pensarsi "nemici".

La ricerca, finalizzata a rilevare le conseguenze di un quartiere di una città di provincia che si difende e si polarizza nei confronti degli stranieri, ha permesso di studiare le prospettive di significato e le pratiche emergenti all'interno dei contesti di vita. È in questo spazio che abbiamo rilevato atteggiamenti, percezioni e azioni che possiamo definire "microradicalizzate". Per esempio, abbiamo analizzato le rappresentazioni distorte e le pratiche disfunzionali di medici, infermieri, professionisti sanitari, impegnati in presidi ospedalieri considerati "di provincia" all'interno di territori sempre più multiculturali. La nostra tesi è che i professionisti sanitari siano "portatori inconsapevoli" di visioni e rappresentazioni distorte e precritiche (Mezirow, 2003) circa la gestione della multietnicità nei luoghi di lavoro, perché generati dentro setting informali spesso monoculturali (Bruni, Fasol, \& Gherardi, 2007). Alcune scelte etnocentriche vengono mascherate sotto forma di universalismo. Dietro la propaganda del "non fare le differenze perchè tutti i pazienti sono uguali", c'è la richiesta implicita di acculturazione alle norme e ai protocolli autoctoni. Sono proprio i reparti dei presidi ospedalieri quelli ad essere maggiormente aggrappati ad una logica di uguaglianza per la quale "non conta se sei straniero, italiano, marocchino, musulmano, islamico, ortodosso, ma conta che sintomo hai, che tipo di paziente sei e quale protocollo si deve applicare e quanto sei capace di adeguarti a queste regole" (medico di ematologia) (Romano, 2020b).

\footnotetext{
${ }^{4}$ Il quartiere come la scuola o i presidi ospedalieri sono arene sociali che possono essere paragonabili a palcoscenici sociali, in cui vengono negoziati i significati utili al vivere insieme, oppure, oppositamente, restare distanti (Goffman, 1971).
} 
Nello studio sul campo, abbiamo incontrato scuole che si stanno svuotando se la presenza di studenti provenienti da altre nazioni supera un livello "soglia". Abbiamo studi di caso in cui i genitori ritirano i propri figli da una scuola dove quel livello "soglia" di presenza diventa per loro intollerabile. Gli stranieri minacciano e ostacolano la carriera scolastica dei figli. I figli si confrontano con una prospettiva di significato e di azione profondamente discriminante. Il compagno con cui giocano a calcio diventa improvvisamente un pericolo per la propria carriera futura (Tab. 1).

Tab. 1 - La scuola vuota (Tratto da Fabbri \& Melacarne, 2020)

Una delle istituzioni pubbliche studiate è una scuola secondaria di primo grado considerata tra le più prestigiose della città. Gli iscritti provenivano da tutti i quartieri. Lì si insegnava il latino, caratteristica che rappresentava una delle forze propulsive dell'espansione della scuola. Per molti genitori era il passaporto per il liceo, una scelta che caratterizzava chi voleva continuare gli studi e acquisire una buona base umanistica. Nell'ultimo decennio, il quartiere in cui è situata la scuola subisce diverse trasformazioni demografiche e la sua composizione è sempre più multietnica. Poi ci sono i rumori, il passaparola di convinzioni personali. Gli alunni stranieri, molti dei quali i genitori hanno la cittadinanza italiana e i loro figli sono nati in Italia, rischiano di far ritardare il programma, non vale la pena di rischiare e iscrivere i propri figli in quel contesto scolastico. La scuola non è più la stessa, è una scuola frequentata troppo da extracomunitari. Nel tempo, molte famiglie, in silenzio, ritirano i figli. Calano drasticamente le iscrizioni. I genitori autoctoni sono assaliti da dubbi e paure. Dubbi e paure però tradotte nella certezza di appartenere a tradizioni culturali superiori rispetto a quelle di cui sono portatori alunni stranieri da cui - si è convinti - non ci sia niente da imparare, perché si tratta di sottoculture. Vivono nei nostri quartieri, i figli frequentano le nostre scuole. Più il contatto diventa stringente più il senso di minaccia aumenta: Così, ci sarà un abbassamento dei livelli di apprendimento dei nostri ragazzi? La presenza così numerosa di alunni stranieri consentirà di svolgere il programma? Come coniugare eccellenza e multiculturalità?

Una figura apicale della scuola ci fornisce una interessante chiave di lettura:

Io sto facendo in quest'ultimo mese le riunioni con i genitori interessati alle iscrizioni e arrivo a questo momento sempre nel timore che l'esito non sia proprio entusiasmante. Viviamo sempre un po' il calo di iscritti soprattutto nella componente italiana degli abitanti del quartiere, che da qui potrebbe scegliere altri istituti scolastici.

Però, quello che posso dire con orgoglio dell'istituto è che è un istituto che continua a lavorare bene per ognuno dei segmenti di popolazione scolastica che ha. Fa un buono, anzi un ottimo lavoro con tutti gli alunni e le alunne. Si tratta di un risultato eccellente, spesso ignorato. Avere il $45 \%$ di alunni di provenienza straniera viene rappresentato come dato saliente rispetto ai dati sulle carriere di studenti e studentesse.

Il $45 \%$ di presenza di studenti stranieri è il dato su cui si costruiscono le rappresentazioni dei genitori, dove ogni discussione è oscurata da questo. Stranieri troppi stranieri. 
Provando a leggere queste affermazioni dal punto di vista dei processi di apprendimento, le paure, i conflitti di interesse e di valore che si incontrano sono la tematizzazione del senso di minaccia che si avverte di fronte a forme di differenza visibili e intrusive. Vivono nei nostri quartieri, i figli frequentano le nostre scuole. Più il contatto diventa stringente più il senso di minaccia aumenta. Quelle in atto negli spazi e nelle pratiche della vita quotidiana, sono forme di microradicalizzazione cognitiva che si caratterizzano per le strategie di rottura che impongono nei diversi contesti sociali, dove l'altro è vissuto come minaccia, alla propria famiglia, alla propria identità, ai propri figli.

\section{La prospettiva trasformativa come framework interpretativo della radicalizzazione}

La letteratura internazionale consente di evidenziare un ampio uso del termine radicalizzazione, che può includere o meno azioni violente indirizzate ad altri gruppi e/o persone, oppure stadi iniziali di radicalizzazione (Bramadat \& Dawson, 2014; Balzacq \& Settoul, 2020). A questo proposito, Amiraux e Araya-Moreno (2014) insistono sul fatto che la maggior parte delle ricerche su questi focus, seppur sofisticate, affrontano il concetto di radicalizzazione in sé come una sorta di patologia che si inserisce in una traiettoria sociale, al termine della quale gli individui, o i gruppi, adotterebbero delle ideologie politico-religiose estreme, giustificando così il passaggio all'atto violento ed il terrorismo (Bramadat \& Dawson, 2014; Balzacq \& Settoul, 2020). Questi approcci, spiegano Amiraux e Araya-Moreno (2014) e Amiraux e Fabbri (2020), non riescono a concepire la radicalizzazione come fenomeno ordinario (o meglio, un micro-fenomeno che risulta da interazione ordinarie) più che straordinario.

A fronte di questo scenario, la prospettiva da cui siamo partiti è stata quella di considerare la radicalizzazione come una modalità di pensiero che possiamo definire precritico (Mezirow, 2003). Questo è appreso in contesti di cattività sociale e culturale attraverso esperienze di contatto fallite, percorsi di vita materiale frustranti, aspettative tradite, bisogni di appartenenza non sostenuti dalle diverse comunità accoglienti. Quali processi si mettono in moto, per esempio, quando in una scuola di prestigio si iscrivono ragazze e ragazzi alloctoni e un numero elevato di genitori tolgono i propri figli "italiani" trasferendoli in contesti "senza stranieri”?

La teoria trasformativa offre una chiave interpretativa di tipo cognitivo per spiegare i diversi livelli di sviluppo del pensiero, andando a spacchettare 
sia come si apprende, sia come l'apprendimento può essere una traiettoria di cambiamento.

Il pensiero precritico è lo stadio del nostro pensare in cui le categorie con cui leggiamo il mondo sono date per scontato e certe, vere ontologicamente, e non storicamente generate. La microradicalizzazione, dentro il quadro della teoria trasformativa (Mezirow, 2003), rappresenta l'inadeguatezza cognitiva a partecipare ad una discussione dialettica o ad affrontare un problema in termini critico-riflessivi, che si fondi non sulle percezioni, ma sui dati e sulle conoscenze che aiutano a comprendere un contesto che a primo contatto può sembrare inadeguato e poco rassicurante (Fabbri \& Melacarne, 2020). La radicalizzazione, in questo senso, è sia una categoria teorica che un fenomeno emergente da pratiche di contatto ad alta densità multietnica, che può essere compreso e spacchettato dentro il campo degli studi sull'apprendimento come fenomeno sociale (Wilner \& Dubouloz, 2015) e dell'apprendimento informale (Watkins \& Marsick, 2020) e trasformativo (Mezirow, 2003).

In questo scenario, l'ipotesi centrale di FORwARD è che le comunità, le organizzazioni e i professionisti debbano essere supportati nell'elaborazione di modelli interpretativi inclusivi, ovvero nell'apprendere nuove prospettive di significato che permettano di sperimentare nuove forme di interazioni e di trasformare i modelli di riferimento adottati da culturalmente assimilati a intenzionalmente assunti (Mezirow, 2003; Taylor \& Cranton, 2012; Amiraux \& Fabbri, 2020).

Se si prende atto che non è possibile contrastare la radicalizzazione solo con misure securitarie (Khosrokhavar, 2017) si apre un territorio che ha a che fare con gli apprendimenti delle comunità, gli apprendimenti delle organizzazioni, gli apprendimenti dei singoli.

La società pone delle sfide che richiedono la produzione di conoscenze utili, spendibili, che, non criminalizzando il pensiero, si pongano il problema dell'affrontamento di processi di radicalizzazione attraverso azioni trasformative. Ognuno di noi è potenzialmente radicalizzato su un aspetto, su un tema, su una posizione. La radicalizzazione è un rischio che tutti corriamo, ed è la consapevolezza di ciò che invita la ricerca a prendere atto del perché alcune conoscenze o azioni sono inutili o fallimentari, e a mettere a fuoco sistemi di azioni capaci di aprire processi di cambiamento possibili.

\section{Acknowledgement}

Il contributo è stato pubblicato all'interno del Progetto FORwARD (ID MUR (85901). Le azioni di ricerca-formazione descritte sono state supportate con il finanziamento previsto dal progetto. 


\section{Riferimenti bibliografici}

Amiraux, V., \& Fabbri, L. (2020). Apprendere a vivere in una società multietnica. Educational Reflective Practices, 1, pp. 5-17.

Amiraux, V., \& Araya-Moreno, J. (2014). Pluralism and radicalization: Mind the gap! Religious Radicalization and Securitization in Canada and Beyond, pp. 92120.

Bailey, G., \& Edwards, P. (2016). Rethinking Radicalization: Microradicalizations and Reciprocal Radicalization as an Intertwined Process. Journal for Deradicalization, 12, pp. 255-281.

Bramadat, P., \& Dawson, L. (2014). Religious Radicalization and Securitization in Canada and Beyond. Toronto: University of Toronto Press.

Balzacq, T., \& Settoul, E. (2021). Radicalization in Theory and Practice: Pathways to National Security in Western Europe. Michigan: Michigan University Press.

Bramadat, P., \& Dawson, L. L. (Eds.) (2014). Religious radicalization and securitization in Canada and beyond. Toronto: University of Toronto Press.

Brookfield, S. D., \& Holst, J. D. (2011). Radicalizing Learning: Adult Education for a Just World. San Francisco, CA: Jossey-Bass.

Bruni, A., Fasol, R., \& Gherardi, S. (2007). L'accesso ai servizi sanitari. Traiettorie, differenze, disuguaglianze. Roma: Carocci Editore.

Byington, B. (2019). Antisemitic Conspiracy Theories and Violent Extremism on the Far Right: A Public Health Approach to Counter-Radicalization. Journal of Contemporary Antisemitism, 2(1), pp. 1-18.

Caramellino, D., Melacarne, C., \& Benjamin, D. (2021). Transformative Learning and Micro-radicalization. In A. Nicolaides, S. Eschenbacher, P. Buerguelt, Y. Gilpin-Jackson, M. Welch, \& M. Misawa (Eds.), Handbook of learning for transformation. New York: Palgrave Macmillan.

Catarci, M., \& Fiorucci, M. (Eds.) (2015). Intercultural Education in the European Context. Theories, Experiences, Challenges. London-New York: Routledge.

Colombo, E. (2020). Sociologia delle relazioni interculturali. Roma: Carocci Editore.

Colombo, E., \& Rebughini, P. (2016). Intersectionality and beyond. Rassegna di sociologia, LVIII(3), pp. 439-460.

Crenshaw, K. (2017). On intersectionality: essential writings. New York: The New Press.

Doosje, B., Moghaddam, F. M., Kruglanski, A. W., de Wolf, A., Mann, L. \& Feddes, A. R. (2016). Terrorism, radicalization and de-radicalization. Current Opinion. Psychology, 11, pp. 79-84.

Fabbri, L., \& Melacarne, C. (2020). Apprendere e disapprendere nei contesti di vita quotidiana. Il caso di un quartiere multietnico. Educational Reflective Practices, 1, pp. 18-54.

Fabbri, L., \& Romano, A. (2017). Metodi per l'apprendimento trasformativo. Casi, modelli, teorie. Milano: Mondadori.

Fiorucci, M., Pinto Minerva, F., \& Portera, A. (a cura di) (2017). Gli alfabeti dell'intercultura. Pisa: ETS. 
Freire, P. (1971). La pedagogia degli oppressi. Milano: Mondadori.

Geeraerts, D. (2012). Cultural models of linguistic standardization. In Cognitive models in language and thought, pp. 25-68. De Gruyter Mouton.

Goffman, E. (1971). Relations in public. Microstudies of the public order. New York, NY: Transaction.

Hearn, J., \& Louvrier, J. (2015). Theories of Diversity and Intersectionality: What Do They Bring to Diversity Management?. In R. Bendl, I. Bleijenbergh, E. Henttonen, \& A.J. Mills (Eds), The Oxford Handbook of Diversity in Organizations. Oxford: Oxford University Press.

Khosrokhavar, F. (2017). Radicalization. Why Some People Choose the Path of Violence. New York: New Press.

Lamberty, P., \& Leiser, D. (2019). Sometimes you just have to go in The link between conspiracy beliefs and political action.

Mantovan, C. (2016). La governance dei quartieri multietnici ad alta conflittualità sociale: il caso dell'area di via Piave a Mestre. Archivio di studi urbani e regionali, XLVII(116), pp. 5-26.

Mezirow, J., \& Taylor, E.W. (Eds.) (2011). Transformative Learning: Theory to Practice. Insights from Community, Workplace, and Higher Education. San Francisco: John Wiley.

Mezirow, J. (1991). Transformative Dimensions of Adult Learning. San Francisco: Jossey-Bass.

Mezirow, J. (2003). Transformative Learning as Discourse. Journal of Transformative Education, 1(1), pp. 58-63.

Pino, M. (2012). Storie di immigrazione: geografia dell'alterità in un quartiere periferico a Milano. Milano: CLUEP.

Ponzo, I. (2009). Abitare al plurale. Differenze e disparità abitative tra gli stranieri. Meridiana, 62, pp. 145-58.

Romano, A. (2020a). Diversity \& Disability Management. Esperienze di inclusione sociale. Milano: Mondadori.

Romano, A. (2020b). Trasformazioni in corsia. Protocolli e pratiche di gestione in contesti sanitari ad alto tasso multietnico. Educational Reflective Practices, 2, pp. 54-85.

Sabic-El-Rayess, A., \& Marsick, V. (2021). Transformative Learning and Extremism. In J. Walker, G. Maestrini, \& S. Smythe (Eds.), Proceedings of the Adult Education in Global Times Conference, pp. 636-638. Ottawa: Canadian Association for the Study of Adult Education (CASAE).

Spivak, G.C. (1999). A Critique of Postcolonial Reason: Toward a History of the Vanishing Present. Harvard: Harvard University Press.

Taylor, E. W., \& Cranton, P. (Eds.) (2012). The handbook of transformative learning: Theory, research, and practice. San Francisco, CA: Jossey-Bass.

Yuval-Davis N. (2006). Intersectionality and Feminist Politics. European Journal of Women's Studies, 13(3), pp. 193-209.

Watkins, K. E., \& Marsick, V. J. (2020). Informal and Incidental Learning in the time of COVID-19. Advances in Developing Human Resources, pp. 1-9. Doi: 10.1177/2F1523422320973656. 
Welsh, R. O., \& Swain, W. A. (2020). (Re)Defining Urban Education: A Conceptual Review and Empirical Exploration of the Definition of Urban Education. Educational Researcher, 49(2), pp. 90-100.

Wilner, A, \& Dubouloz, C.J. (2015). Homegrown terrorism and transformative learning: an interdisciplinary approach to understanding radicalization. Global Change, Peace, and Security, 22(1), pp. 33-51.

Yang, J. (2012). An overview of building learning cities as a strategy for promoting lifelong learning. Journal of Adult and Continuing Education, 18(2), pp. 97-113. 